\title{
New Freezeout Mechanism for Strongly Interacting Dark Matter
}

\author{
Juri Smirnov $\oplus^{1,2, *}$ and John F. Beacom $\oplus^{1,2,3, \dagger}$ \\ ${ }^{1}$ Center for Cosmology and AstroParticle Physics (CCAPP), The Ohio State University, Columbus, Ohio 43210, USA \\ ${ }^{2}$ Department of Physics, The Ohio State University, Columbus, Ohio 43210, USA \\ ${ }^{3}$ Department of Astronomy, The Ohio State University, Columbus, Ohio 43210, USA
}

(Received 27 February 2020; revised 6 July 2020; accepted 10 August 2020; published 25 September 2020; corrected 29 September 2020)

\begin{abstract}
We present a new mechanism for thermally produced dark matter, based on a semi-annihilation-like process, $\chi+\chi+\mathrm{SM} \rightarrow \chi+\mathrm{SM}$, with intriguing consequences for the properties of dark matter. First, its mass is low, $\lesssim 1 \mathrm{GeV}$ (but $\gtrsim 5 \mathrm{keV}$ to avoid structure-formation constraints). Second, it is strongly interacting, leading to kinetic equilibrium between the dark and visible sectors, avoiding the structureformation problems of $\chi+\chi+\chi \rightarrow \chi+\chi$ models. Third, in the $3 \rightarrow 2$ process, one dark matter particle is consumed, giving the standard-model particle a monoenergetic recoil. We show that this new scenario is presently allowed, which is surprising (perhaps a "minor miracle"). However, it can be systematically tested by novel analyses in present and near-term experiments. In particular, the Co-SIMP model for thermal-relic dark matter can explain the XENON1T excess.
\end{abstract}

DOI: 10.1103/PhysRevLett.125.131301

Introduction.-For dark matter (DM) models, thermal production mechanisms are highly predictive frameworks [1-3]. Charting possible realizations is important, as it leads to insights that guide experimental efforts to fully test particle dark matter. A thermal production process, if confirmed experimentally, would provide a new probe of the physical conditions of the early Universe.

The best studied thermal candidate is the weakly interacting massive particle (WIMP) [4-8]. In the simplest case, the annihilation cross section to all final states is determined from the relic abundance as $\langle\sigma v\rangle=$ $\left(2.2 \times 10^{-26} \mathrm{~cm}^{3} \mathrm{~s}^{-1}\right)\left(0.12 / \Omega_{\mathrm{DM}} h^{2}\right)$ [9]. The "WIMP window" is defined by the smallest mass allowed by annihilation constraints $(20 \mathrm{GeV}$ if neutrinos are neglected [10]; $10 \mathrm{MeV}$ if they are dominant [11]) and the largest mass allowed by unitarity $(150 \mathrm{TeV}[12,13])$.

It is important to consider other possibilities [14-25]. Recent work $[18,25]$ has made the simple but ingenious point that the process $\chi+\chi+\chi \rightarrow \chi+\chi$ is efficient in the early Universe if the interactions are strong, setting the relic abundance while involving only dark-matter processes, hence the name strongly interacting massive particle (SIMP). The observed DM abundance requires $\left\langle\sigma_{32} v_{\text {rel }}^{2}\right\rangle M_{\mathrm{DM}}^{2} \approx$ $10^{8} \mathrm{GeV}^{-3}$ [18,26]. Assuming a scaling behavior of $\left\langle\sigma_{32} v_{\text {rel }}^{2}\right\rangle \equiv \alpha_{\text {eff }}^{3} / M_{\mathrm{DM}}^{5}$ implies an MeV-scale dark matter

Published by the American Physical Society under the terms of the Creative Commons Attribution 4.0 International license. Further distribution of this work must maintain attribution to the author(s) and the published article's title, journal citation, and DOI. Funded by SCOAP. mass [18]. A dark matter sector that converts DM rest mass into kinetic energy that is kinetically decoupled from the standard model (SM) will heat itself up [27]. The DM freestreaming length would then be too long, as the DM particles would be too fast. To dissipate the heat into the SM sector and to slow down the DM particles, an elastic SM-SIMP interaction, $\chi+\mathrm{SM} \rightarrow \chi+\mathrm{SM}$, has to be postulated [18]. However, in the mass range relevant for the SIMPs, it is hard to do that without inducing new $\chi+\chi \rightarrow \chi+\chi$ interactions $[25,28]$ that conflict with cluster observations [29-32] (though there may be ways out [33-37]).

We take a different approach and suppose that the DM decouples through $\chi+\chi+\mathrm{SM} \rightarrow \chi+\mathrm{SM}$. Figure 1 shows this number-changing interaction for the DM, which also keeps it in kinetic equilibrium with the SM plasma, avoiding overheating the DM. A $Z_{3}$ symmetry guarantees DM stability and leads to the dominance of the above interaction. As detailed below, what we call the Co-SIMP mechanism leads to a dark-matter candidate with vastly different properties and phenomenology from other thermal relics. Despite the Co-SIMP's low mass and strong

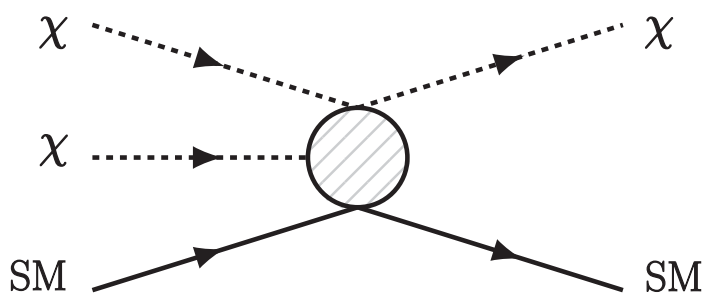

FIG. 1. The Co-SIMP freezeout process, which also keeps DM in kinetic equilibrium with the SM. This process gives novel monoenergetic-recoil signals in detectors. 
interactions, it seems to have evaded all present constraints. However, testing Co-SIMPs is within reach.

In the following, we define the Co-SIMP model, calculate the corresponding freezeout process and its consequences, then predict present-day signals based on Fig. 1, as well as signals expected from loop-induced elastic interactions, and conclude.

Co-SIMP interactions. - Given the Co-SIMP interaction in Fig. 1, the dark sector must have a $Z_{3}$ symmetry (this could be generalized to $Z_{N}$ with $N>3$ ) to ensure DM stability, similar to Ref. [15]. To prevent a WIMP-like freezeout $(\chi+\chi \rightarrow \chi+\mathrm{SM}+\mathrm{SM})$, we require $M_{\mathrm{DM}} \leq 2 M_{\mathrm{SM}}$. Direct couplings to photons or neutrinos are prohibited to avoid $2 \rightarrow 3$ processes such as $\chi+\chi \rightarrow \chi+\gamma+\gamma$. The final state of the freezeout process is, for $M_{\mathrm{DM}} \ll M_{\mathrm{SM}}$, a semirelativistic $\chi$ and a nonrelativisitic SM particle. For the lower mass bound, we take $M_{\mathrm{DM}}>5 \mathrm{keV}$ to avoid structure-formation constraints [38,39]. More specific constraints are discussed below.

The Co-SIMP upper mass bound varies depending on the DM interaction operator. For a leptophilic model, coupled via $\mathcal{O}_{\ell}=\bar{\ell}_{\alpha} \ell_{\beta} \chi^{3} / \Lambda^{2}$, we require $M_{\mathrm{DM}} \lesssim \mathrm{MeV}$. For a nucleophilic model coupled via $\mathcal{O}_{n}=\bar{n} n \chi^{3} / \Lambda^{2}, \mathcal{O}_{p}=$ $\bar{p} p \chi^{3} / \Lambda^{2}$, or $\mathcal{O}_{\pi}=\pi^{2} \chi^{3} / \Lambda$, we require $M_{\mathrm{DM}} \lesssim \mathrm{GeV}$. We focus on the leptophilic and nucleophilic scenarios. (A more exotic possibility arises if coupling to weak gauge bosons via $\mathcal{O}_{W}=F_{\mu \nu}^{W} F^{W \mu \nu} \chi^{3} / \Lambda^{3}$, or top quarks only, is allowed, in which case the DM mass can be as large as $\sim 100 \mathrm{GeV}$. In the case of coupling to $W$ bosons, a particular construction of the UV completion is required in order to avoid direct photon couplings).

Our focus is on defining a new framework for thermal DM; see the Supplemental Material [40] for further exploration of constraints, as well as possible UV completions, which are crucial for investigating Co-SIMP interactions at colliders and other high-energy environments.

Co-SIMP freezeout.-The Boltzmann equation for freezeout via $\chi+\chi+\mathrm{SM} \rightarrow \chi+\mathrm{SM}$ is

$$
\begin{aligned}
s H(T) z Y_{\mathrm{DM}}^{\prime} & =-\gamma_{32}\left(\frac{n_{\mathrm{DM}}^{2}}{n_{\mathrm{DM}, \mathrm{eq}}^{2}}-\frac{n_{\mathrm{DM}}}{n_{\mathrm{DM}, \mathrm{eq}}}\right) \\
& =-\left\langle\sigma_{32} v_{\mathrm{rel}}^{2}\right\rangle\left(n_{\mathrm{DM}}^{2}-n_{\mathrm{DM}} n_{\mathrm{DM}, \mathrm{eq}}\right) n_{\mathrm{SM}, \mathrm{eq}} .
\end{aligned}
$$

The space-time interaction density is approximated as $\gamma_{32} \approx$ $\left\langle\sigma_{32} v_{\text {rel }}^{2}\right\rangle n_{\mathrm{DM}, \mathrm{eq}}^{2} n_{\mathrm{SM} \text {,eq }}$ in the nonrelativistic regime. The Hubble rate is $H(T)$ and $s$ the entropy density. Defining $z=M_{\mathrm{DM}} / T$ and $Y_{\mathrm{DM}}^{\prime}=d Y_{\mathrm{DM}} / d z$,

$$
Y_{\mathrm{DM}}^{\prime}=-\frac{\lambda_{32}}{z^{5}}\left(Y_{\mathrm{DM}}^{2}-Y_{\mathrm{DM}} Y_{\mathrm{DM}, \mathrm{eq}}\right) .
$$

The dimensionless quantities are

$$
\lambda_{32}=\left(Y_{\mathrm{SM}}^{\mathrm{sym}}+\eta\right) \times\left.\frac{\left\langle\sigma_{32} v_{\mathrm{rel}}^{2}\right\rangle s^{2}}{H(T)}\right|_{M_{\mathrm{DM}}},
$$

with $Y_{\mathrm{DM}}=n_{\mathrm{DM}} / s, Y_{\mathrm{SM}}^{\mathrm{sym}}=n_{\mathrm{SM}}^{\mathrm{sym}} / s$ being the symmetric SM particle abundance, and $\eta$ the baryon-to-photon ratio. Application of the boundary-layer method [26,98] yields the asymptotic relic abundance

$$
Y_{\mathrm{DM}}(\infty) \approx-\left(\int_{z_{\text {f.o. }}}^{\infty} \frac{\lambda_{32}}{z^{5}} d z\right)^{-1} .
$$

The freezeout temperature is defined by $z_{\text {f.o. }} \approx \log \lambda_{32}-$ $5 / 2 \log z_{\text {f.o. }}-1.65$, which gives $z_{\text {f.o. }} \approx 10$. Note the dependence of the interaction factor on $v_{\text {rel }}^{2}$, which results from an incoming flux of two particles on target. At leading order, in exothermic processes $\sigma_{32} v_{\text {rel }}^{2} \sim$ const [17]. The loop-induced process $\chi \chi \rightarrow \chi+2 \gamma$ is subdominant over the Co-SIMP parameter space (its impact in the late universe is discussed in the Supplemental Material [40]).

The relic density is $\Omega_{\mathrm{DM}} \approx Y_{\mathrm{DM}}(\infty) M_{\mathrm{DM}} s_{0} / \rho_{\text {crit }}$. For the typical case, $M_{\mathrm{DM}} \ll M_{\mathrm{SM}}$, the cross section of the number-changing interaction is

$$
\left\langle\sigma_{32} v_{\text {rel }}^{2}\right\rangle_{\text {f.o. }} \approx 10^{12}\left(\frac{10^{-9}}{\eta}\right)\left(\frac{\mathrm{MeV}}{M_{\mathrm{DM}}}\right)^{3}\left(\frac{0.12}{\Omega_{\mathrm{DM}} h^{2}}\right) \mathrm{GeV}^{-5} .
$$

For the edge case, $M_{\mathrm{DM}} \approx M_{\mathrm{SM}}$, the decoupling of Co-SIMP interactions takes place at higher temperatures, such that the Co-SIMP freezeout happens dominantly through interactions with the symmetric component of the SM particle bath, leading to

$\left\langle\sigma_{32} v_{\text {rel }}^{2}\right\rangle_{\text {f.o. }} \approx 5 \times 10^{3}\left(\frac{10 \mathrm{MeV}}{M_{\mathrm{DM}}}\right)^{3}\left(\frac{0.12}{\Omega_{\mathrm{DM}} h^{2}}\right) \mathrm{GeV}^{-5}$.

These are the central predictions of the new Co-SIMP thermal production mechanism.

Considerations related to light-element dissociation during big bang nucleosynthesis (BBN) [99-101] do not constrain Co-SIMPs, because the energy released into SM particles is much smaller than for WIMP freezeout. However, in the leptophilic case, because the Co-SIMP can have $M_{\mathrm{DM}} \ll m_{e}$, the number of relativistic degrees of freedom $N_{\text {eff }}$ is affected, in fact being reduced from the standard value. We discuss this in detail in the Supplemental Material [40], considering both BBN and cosmic microwave background (CMB) observations. Because of this negative contribution, if experiments find a Co-SIMP with a small mass, it would indicate a complex early history. For example, a cancellation with a positive contribution to $\Delta N_{\text {eff }}$ could take place to explain the current data, which are consistent with $\Delta N_{\text {eff }} \approx 0$ [102].

In the nucleophilic case, the Co-SIMP masses are above the $\mathrm{MeV}$ scale, so that BBN observables and $\Delta N_{\text {eff }}$ are not affected.

Testing the Co-SIMP process.-Strikingly, the Co-SIMP freezeout process $(\chi+\chi+\mathrm{SM} \rightarrow \chi+\mathrm{SM})$ happens directly in detectors with a significant rate, unlike for WIMPs $(\chi+\chi \rightarrow \mathrm{SM}+\mathrm{SM})$ [103]. This is possible because of the 
strong DM interactions, the high DM density due to the low DM mass, and the high SM density of ordinary materials.

The $3 \rightarrow 2$ process produces energetic SM particles in a detector, like WIMP direct detection, but with important differences. First, the kinetic energy of the SM particle, provided by the consumption of one DM particle, is monoenergetic. This follows directly from the fact that in the $\mathrm{CM}$ frame, the outgoing particles have equal momenta. The SM recoil energy is

$$
E_{R} \approx \frac{3 M_{\mathrm{DM}}^{2}\left(M_{\mathrm{DM}}+2 M_{\mathrm{SM}}\right)\left(3 M_{\mathrm{DM}}+2 M_{\mathrm{SM}}\right)}{8 M_{\mathrm{SM}}\left(2 M_{\mathrm{DM}}+M_{\mathrm{SM}}\right)^{2}},
$$

which reduces to $E_{R} \approx 3 / 2 M_{\mathrm{DM}}^{2} / M_{\mathrm{SM}}$ (note $E_{R} \ll M_{\mathrm{DM}}$ ) for the typical case, $M_{\mathrm{DM}} \ll M_{\mathrm{SM}}$, and to $E_{R} \approx 5 / 8 M_{\mathrm{DM}}$ for the edge case, $M_{\mathrm{DM}} \approx 2 M_{\mathrm{SM}}$. The kinetic energy of the incoming DM particle is negligible in this reaction. Second, though the kinetic energy of the SM particle will be at most barely relativistic, it can be well above the energy produced by a recoiling WIMP of comparable mass. For the WIMP case, the recoil energy is $E_{R} \approx 2 v^{2} \mu^{2} / M_{\mathrm{SM}}$, with $\mu$ being the reduced mass [104], which is a factor $v^{2} \sim 10^{-6}$ smaller than for the Co-SIMP case. Those two points make for a signal that could stand out from backgrounds. (Other scenarios with large energy deposits involve DM deexcitation [105-109].) Third, even if elastic scatterings in the detector overburden slow the Co-SIMPs down, the expected signal spectrum shape is unaltered because it depends on the loss of DM rest mass, not kinetic energy.

The event rate per detector volume is

$$
\begin{aligned}
\gamma_{32} & =\frac{R}{V} \approx\left\langle\sigma_{32} v_{\mathrm{rel}}^{2}\right\rangle n_{\mathrm{DM}}^{2} n_{\mathrm{SM}} \\
& \approx \frac{0.8}{\mathrm{~m}^{3} \text { day }}\left(\frac{n_{\mathrm{SM}}}{N_{A} \mathrm{~cm}^{-3}}\right)\left(\frac{0.1 \mathrm{MeV}}{M_{\mathrm{DM}}}\right)^{2}\left(\frac{\left\langle\sigma_{32} v_{\mathrm{rel}}^{2}\right\rangle}{10^{16} \mathrm{GeV}^{-5}}\right),
\end{aligned}
$$

where $n_{\mathrm{SM}}$ and $n_{\mathrm{DM}}$ are the SM and DM number densities, $N_{A}$ is the Avogadro number, and we use the observed DM mass density.

Figure 2 shows the monoenergetic spectrum in XENON1T caused by the leptophilic Co-SIMP process. We include energy resolution, which is 3.5\% in this energy range [110]. Superposed are their measured electron recoil data. This shows that a bump hunt could be highly efficient for testing the Co-SIMP scenario.

Figure 3 shows the Co-SIMP parameter space, including current constraints. For the Borexino experiment [111], we convolve the predicted signal with the energy resolution and compare it to the measured data in Ref. [112]. The uncertainty scale is set by the square root of the number of measured events in a bin of width somewhat larger than the energy resolution. This is appropriate because the backgrounds are well modeled. For XENON1T, we have an analogous procedure. We use data from their double electron capture (DEC) search [110] and their S2-only light

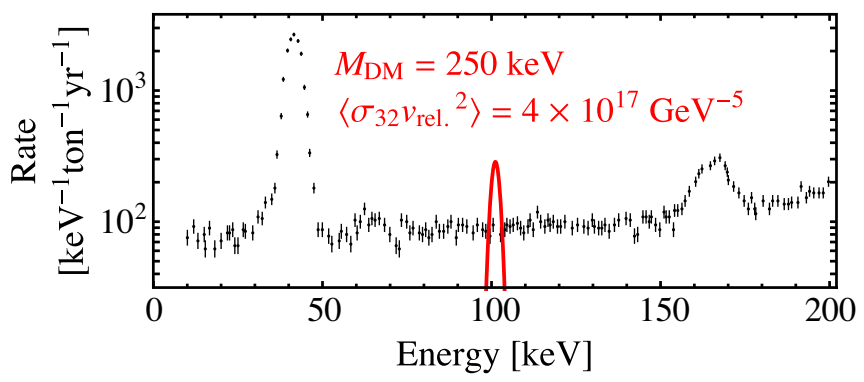

FIG. 2. Monoenergetic electron recoil spectrum caused by the Co-SIMP process for $M_{\mathrm{DM}}=250 \mathrm{keV}$ (hence $E_{R} \approx 101 \mathrm{keV}$ ), including energy resolution, compared to XENON1T data. For better visibility, $\left\langle\sigma_{32} v_{\text {rel }}^{2}\right\rangle$ is chosen so that the signal is 10 times greater than needed for a $90 \%$ C.L. exclusion $(1.28 \sigma)$.

DM search [113]. At intermediate energies, we use data from their electron recoil study [114], conservatively requiring that the signal be less than the measured events in a bin because the background analysis is still preliminary. (In the Supplemental Material [40], we repeat the analysis taking into account the xenon orbital effects, which weakens the bounds somewhat for low Co-SIMP masses).

Figure 3 also shows the projected sensitivity for 1 ton-yr of XENON1T, assuming a dedicated line search, with well-modeled backgrounds, in their entire electron recoil energy range. Similarly, we show the sensitivity of the proposed DARWIN experiment in 200 ton-yr [115]. Other relevant experiments to search for the Co-SIMP process are KamLAND [116] and JUNO [117].

Figure 4 shows the parameter space for the nucleophilic scenario. It is challenging to test the nucleophilic Co-SIMP process directly in large volume detectors due to lower event rates at larger DM masses. However, loop-induced elastic interactions, discussed below, are efficient for testing nucleophilic interactions.

Elastic interactions.-Figure 5 shows that an elastic CoSIMP scattering process is induced at the two-loop level: $\mathrm{SM}+\chi \rightarrow \mathrm{SM}+\chi$. Given, for example, the electrophilic $\mathcal{O}_{\ell}$ interaction operator, the induced coupling coefficient $c_{\mathrm{e}}^{d}$ of the interaction $\chi \chi \bar{e} e$ can be computed. After performing the top loop integral, one obtains the following expression (with $x=\sqrt{1-4 M_{\mathrm{DM}}^{2} / k^{2}}$ ), which is regularized with a cutoff at the scale $\Lambda$ and analytically approximated, with $M_{\mathrm{SM}}$ being the mass of the propagating electron:

$$
\begin{aligned}
c_{\mathrm{e}}^{d} & =\frac{M_{\mathrm{SM}}}{(4 \pi)^{2} \Lambda^{4}} \int \frac{d^{4} k}{(2 \pi)^{4}} \log \left(\frac{x+1}{x-1}\right)\left(\frac{x}{k^{2}-M_{\mathrm{SM}}^{2}}\right) \\
& \approx \frac{M_{\mathrm{SM}}}{(4 \pi)^{4} \Lambda^{2}}\left(1-\frac{M_{\mathrm{DM}}^{2}}{\Lambda^{2}}\right) \log \left(\frac{\Lambda^{2}+M_{\mathrm{SM}}^{2}}{4 M_{\mathrm{DM}}^{2}}\right) .
\end{aligned}
$$

To make connection to the freezeout process, we compute the $3 \rightarrow 2$ cross section, given the electron Co-SIMP coupling operator $\mathcal{O}_{\ell}$, following Ref. [17], and obtain $\left\langle\sigma_{32} v_{\mathrm{rel}}^{2}\right\rangle \approx \sqrt{3} /\left(4 \pi M_{\mathrm{DM}} \Lambda^{4}\right)$. We can now express the loop 


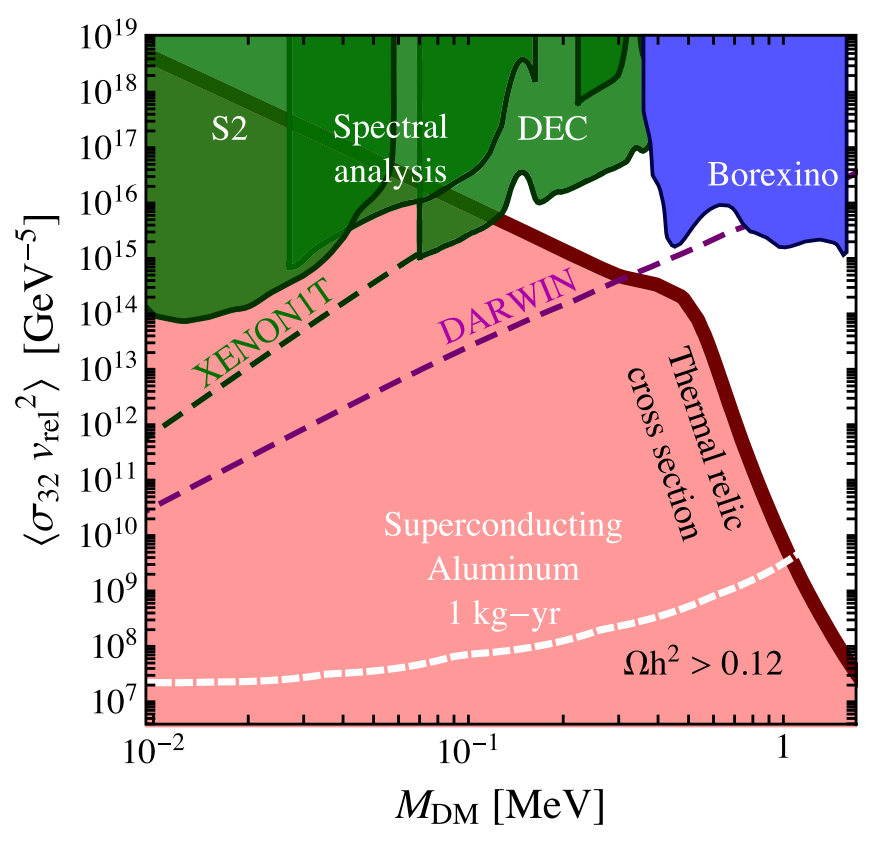

FIG. 3. Current bounds and projected sensitivities for electrophilic Co-SIMPs. The parameter space is largely open, despite the low DM mass and strong couplings. The relic density at $M_{\mathrm{DM}} \ll$ $m_{e}$ is affected by the baryon asymmetry, leading to a change of the slope. We show bounds based on the Co-SIMP process in detectors (colored regions), along with projected sensitivities (dashed lines). A low-threshold detector based on superconducting aluminum could test nearly all the parameter space (white dashed line). In the pink region, the relic density is too high; at its boundary, it is correct.

coefficient as a function of the $3 \rightarrow 2$ cross section, which yields $\left(c_{\mathrm{e}}^{d} \mathrm{GeV}\right) \approx 5 \times 10^{-9} \sqrt{M_{\mathrm{DM}}\left\langle\sigma_{32} v_{\text {rel }}^{2}\right\rangle \mathrm{GeV}^{4}}$. This leads to DM-electron scattering cross sections of $\sigma_{\mathrm{SI}}^{\mathrm{el}} \approx$ $10^{-37} \mathrm{~cm}^{2}$ at $M_{\mathrm{DM}} \sim 100 \mathrm{keV}$ and $\sigma_{\mathrm{SI}}^{\mathrm{el}} \approx 10^{-40} \mathrm{~cm}^{2}$ at $M_{\mathrm{DM}} \sim \mathrm{MeV}$. Those cross section values are currently unconstrained by direct detection experiments, but are within the reach of future experimental efforts [118-120].

Figure 3 shows the expected sensitivity of a low threshold detector to the electrophilic scenario. An efficient technology seems to be detectors based on superconducting materials, for example, aluminum [121,122] (here with $1 \mathrm{~kg}$-yr). Two other technologies for direct detection experiments with a low energy threshold $[119,120]$ are discussed in the Supplemental Material [40].

Figure 4 shows current constraints on the nucleophilic Co-SIMP scenario from a gram-scale cryogenic calorimeter experiment [123]. In addition, we find that a detector based on superfluid helium [124-126], could test a significant fraction of the open parameter space, (here with $1 \mathrm{~kg}-\mathrm{yr}$ and a threshold of $\sim \mathrm{meV}$ ). For an EFT approach to superfluid helium DM sensitivity see Refs. [127-129]. Other techniques, based on polar materials $[130,131]$ could provide comparable sensitivity to nucleophilic Co-SIMPs; see Ref. [132] for an overview of possible targets. The loop-

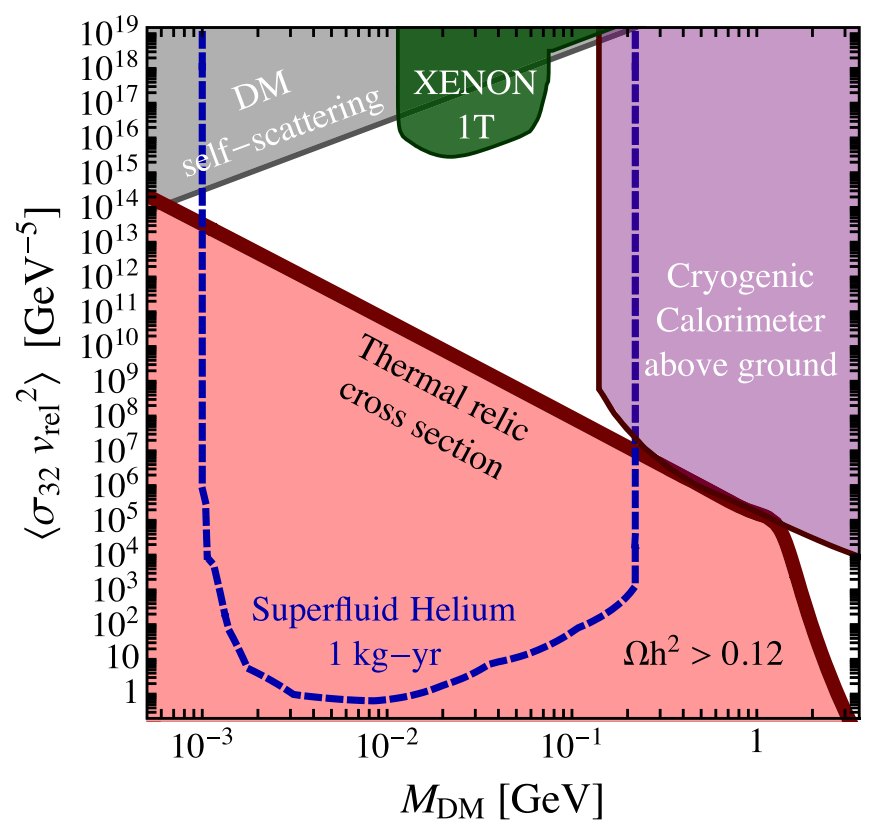

FIG. 4. Current bounds and projected sensitivities for nucleophilic Co-SIMPs. The change in the relic abundance slope is now related to the proton mass. Limits from the XENON1T experiment constrain the Co-SIMP process directly (dark green region). A detector based on superfluid helium could test a large fraction of the currently viable parameter space (blue dashed line).

induced elastic cross section is computed analogously to the leptophilic case, but where the larger mass increases the numerator.

Other possible searches.-In the Supplemental Material [40], we discuss a variety of other constraints on Co-SIMPs, none of which are yet as strong and secure as those above. The Co-SIMP process and loop-induced processes can produce $\mathrm{x}$-rays, but we find that the current sensitivities of X-ray satellites $[133,134]$ are insufficient to be competitive with direct-detection searches. However, future missions, such as Athena [135], will test relevant parameter space. A variant of the Co-SIMP process, $e^{+}+e^{-} \rightarrow \chi+\chi+\chi$, could cool supernovae and presupernova stars [136,137], but its effectiveness is limited by an accompanying opacity due to $\chi+e \rightarrow \chi+\chi+e$. We estimate the Co-SIMP self-scattering elastic cross section induced at two loops, but we find that it is low in the majority of the parameter space and consistent with

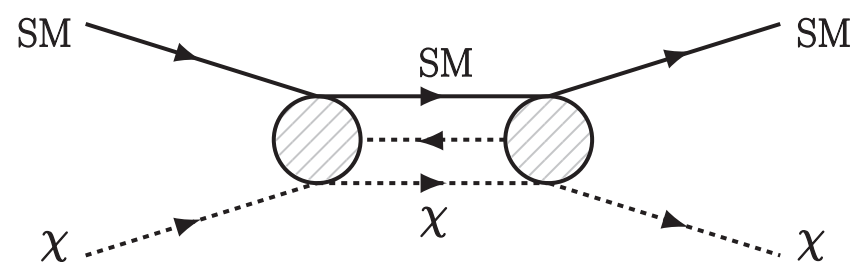

FIG. 5. Elastic dark matter scattering process with SM particles, induced at the two-loop level. 
observations [29-32]. (However, in the nucleophilic scenario, it seems to provide the strongest bound on Co-SIMPs with low masses.) Finally, spectroscopy of SM bound states [138] could test the Co-SIMP. Particularly, true muonium $[139,140]$, with a very compact wave function, would be ideal to test the leptophilic scenario [141], once experimental observations can be performed.

Above, we focus on the most model-independent tests of the Co-SIMP model, i.e., those most closely connected to the Co-SIMP production mechanism and at comparable or lower $\sqrt{s}$ to freezeout. In future work, it would be interesting to explore constraints from higher-energy interactions, though this would require first specifying the UV completion of our framework.

A key question is if or how Co-SIMPs are constrained by collider missing-energy searches, which are generally quite powerful [142-144]. Those searches will be ineffective if the produced Co-SIMPs interact with cross sections larger than $\sigma \sim 10-30 \mathrm{~cm}^{2}$ and thus do not lead to a missing energy signature [145]. Thus, we estimate the semielastic scattering cross section $\sigma_{\chi+e \rightarrow \chi}+\chi+e \approx M_{\mathrm{DM}} \sqrt{s} /$ $\left(256 \pi^{3} \Lambda^{4}\right) \approx \sqrt{s} M_{\mathrm{DM}}^{2}\left\langle\sigma_{32} v_{\text {rel }}^{2}\right\rangle /\left(64 \pi^{2} \sqrt{3}\right)$, where we used the previously derived relation between the effective operator scale and the interaction cross section. For $M_{\mathrm{DM}} \ll m_{e}$ this leads to $\sigma_{\chi+e \rightarrow \chi+\chi+e} \approx 5 \times 10^{-26}$ $\sqrt{E_{\chi} /(100 \mathrm{GeV})} \mathrm{cm}^{2}$ and for $M_{\mathrm{DM}} \approx m_{e}$ to $\sigma_{\chi+e \rightarrow \chi+\chi+e} \approx$ $10^{-28} \sqrt{E_{\chi} /(100 \mathrm{GeV})} \mathrm{cm}^{2}$. For the nucleophilic case, the cross sections are about an order of magnitude larger since $\sqrt{s}$ is bigger. Thus, even if Co-SIMPs are copiously produced, as expected, existing bounds do not apply, and new analyses would be needed.

A related question concerns cosmic-ray interactions with DM, as in Refs. [146-150], where large cross sections for light dark matter were ruled out, at least under the assumption of energy-independent elastic interactions (see Ref. [150] for a model with energy dependence). Constraints for Co-SIMPs will depend on the UV completion, and are reserved for future work.

Conclusions. - It is of high importance to point out thermal production mechanisms of DM, since they lead to highly predictive models and could provide additional information about the conditions of the early Universe. We present a new possibility to thermally produce DM. The new ingredient is that the production mechanism predicts the cross section for a process that consumes one DM particle and converts its rest mass into kinetic energy of the catalyzing SM particle.

This mechanism leads to novel signatures, such as through the Co-SIMP freezeout process occurring in detectors, including large ones for neutrinos. At loop level, an elastic scattering process is induced that can be tested in conventional dark matter detectors. Large fractions of the parameter space remain still untested but seem within reach of future dark matter searches, especially through expected sensitivity improvements in the coming years.

We are grateful for helpful discussions with Nicole Bell, Eric Braaten, Christopher Cappiello, Basudeb Dasgupta, Jared Evans, Yonit Hochberg, Francesco Sannino, Maxim Pospelov, Bei Zhou, Jure Zupan, Kathryn Zurek, and especially Eric Kuflik, and Teresa Marrodan Undagoitia. J. S. is primarily supported by a Feodor Lynen Fellowship from the Alexander von Humboldt Foundation. The work of J.F.B. was supported by NSF Grant No. PHY-1714479.

*smirnov.9@osu.edu

†beacom.7@osu.edu

[1] E. W. Kolb and M. S. Turner, The early Universe, Front. Phys. 69, 1 (1990).

[2] G. Jungman, M. Kamionkowski, and K. Griest, Supersymmetric dark matter, Phys. Rep. 267, 195 (1996).

[3] G. Bertone, D. Hooper, and J. Silk, Particle dark matter: Evidence, candidates and constraints, Phys. Rep. 405, 279 (2005).

[4] Y. Zeldovich, Survey of modern cosmology, Adv. Astron. Astrophys. 3, 241 (1965).

[5] B. W. Lee and S. Weinberg, Cosmological Lower Bound on Heavy Neutrino Masses, Phys. Rev. Lett. 39, 165 (1977).

[6] G. Steigman and M. S. Turner, Cosmological constraints on the properties of weakly interacting massive particles, Nucl. Phys. B253, 375 (1985).

[7] G. Arcadi, M. Dutra, P. Ghosh, M. Lindner, Y. Mambrini, M. Pierre, S. Profumo, and F. S. Queiroz, The waning of the WIMP? A review of models, searches, and constraints, Eur. Phys. J. C 78, 203 (2018).

[8] L. Roszkowski, E. M. Sessolo, and S. Trojanowski, WIMP DM candidates and searches-Current status and future prospects, Rep. Prog. Phys. 81, 066201 (2018).

[9] G. Steigman, B. Dasgupta, and J. F. Beacom, Precise relic WIMP abundance and its impact on searches for DM annihilation, Phys. Rev. D 86, 023506 (2012).

[10] R. K. Leane, T. R. Slatyer, J. F. Beacom, and K. C. Y. Ng, GeV-scale thermal WIMPs: Not even slightly ruled out, Phys. Rev. D 98, 023016 (2018).

[11] K. M. Nollett and G. Steigman, BBN and the CMB constrain neutrino coupled light WIMPs, Phys. Rev. D 91, 083505 (2015).

[12] Kim Griest and Marc Kamionkowski, Unitarity Limits on the Mass and Radius of DM Particles, Phys. Rev. Lett. 64, 615 (1990).

[13] J. Smirnov and J. F. Beacom, TeV-scale thermal WIMPs: Unitarity and its consequences, Phys. Rev. D 100, 043029 (2019).

[14] A. D. Dolgov, New old mechanism of dark matter burning, arXiv:1705.03689.

[15] F. D'Eramo and J. Thaler, Semi-annihilation of dark matter, J. High Energy Phys. 06 (2010) 109. 
[16] M. Farina, D. Pappadopulo, J. T. Ruderman, and G. Trevisan, Phases of cannibal dark matter, J. High Energy Phys. 12 (2016) 039.

[17] J. M. Cline, H. Liu, T. Slatyer, and W. Xue, Enabling forbidden dark matter, Phys. Rev. D 96, 083521 (2017).

[18] Y. Hochberg, E. Kuflik, T. Volansky, and J. G. Wacker, Mechanism for Thermal Relic Dark Matter of Strongly Interacting Massive Particles, Phys. Rev. Lett. 113, 171301 (2014).

[19] U. K. Dey, T. N. Maity, and T. S. Ray, Light dark matter through assisted annihilation, J. Cosmol. Astropart. Phys. 03 (2017) 045.

[20] U. K. Dey, T. N. Maity, and T. S. Ray, Boosting assisted annihilation for a cosmologically safe meV scale dark matter, Phys. Rev. D 99, 095025 (2019).

[21] T. N. Maity and T. S. Ray, Resonant assisted annihilation, J. Cosmol. Astropart. Phys. 11 (2019) 033.

[22] R. T. D’Agnolo, C. Mondino, J. T. Ruderman, and P.-J. Wang, Exponentially light dark matter from coannihilation, J. High Energy Phys. 08 (2018) 079.

[23] H. Kim and E. Kuflik, Super heavy thermal dark matter, arXiv:1906.00981.

[24] R. T. D’Agnolo, D. Pappadopulo, J. T. Ruderman, and P. J. Wang, Thermal relic targets with exponentially small couplings, arXiv:1906.09269.

[25] Y. Hochberg, E. Kuflik, H. Murayama, T. Volansky, and J. G. Wacker, Model for Thermal Relic Dark Matter of Strongly Interacting Massive Particles, Phys. Rev. Lett. 115, 021301 (2015).

[26] N. A. Dondi, F. Sannino, and J. Smirnov, The thermal history of composite dark matter, arXiv:1905.08810.

[27] M. A. Buen-Abad, R. Emami, and M. Schmaltz, Cannibal dark matter and large scale structure, Phys. Rev. D 98, 083517 (2018).

[28] M. Hansen, K. Langæble, and F. Sannino, SIMP model at NNLO in chiral perturbation theory, Phys. Rev. D 92, 075036 (2015).

[29] A. Robertson, R. Massey, and V. Eke, What does the Bullet Cluster tell us about self-interacting dark matter? Mon. Not. R. Astron. Soc. 465, 569 (2017).

[30] Richard Massey et al., The behavior of dark matter associated with four bright cluster galaxies in the $10 \mathrm{kpc}$ core of Abell 3827, Mon. Not. R. Astron. Soc. 449, 3393 (2015).

[31] F. Kahlhoefer, K. Schmidt-Hoberg, J. Kummer, and S. Sarkar, On the interpretation of dark matter selfinteractions in Abell 3827, Mon. Not. R. Astron. Soc. 452, L54 (2015).

[32] S. Y. Kim, A. H. G. Peter, and D. Wittman, In the wake of dark giants: New signatures of dark matter self interactions in equal mass mergers of Galaxy clusters, Mon. Not. R. Astron. Soc. 469, 1414 (2017).

[33] E. Braaten, D. Kang, and R. Laha, Production of darkmatter bound states in the early universe by three-body recombination, J. High Energy Phys. 11 (2018) 084.

[34] H. M. Lee and M.-S. Seo, Communication with SIMP dark mesons via Z'-portal, Phys. Lett. B 748, 316 (2015).

[35] S.-M. Choi and H. M. Lee, SIMP dark matter with gauged $\mathrm{Z}_{3}$ symmetry, J. High Energy Phys. 09 (2015) 063.

[36] S.-M. Choi and H. M. Lee, Resonant SIMP dark matter, Phys. Lett. B 758, 47 (2016).
[37] S.-M. Choi, H. M. Lee, P. Ko, and A. Natale, Resolving phenomenological problems with stronglyinteractingmassive-particle models with dark vector resonances, Phys. Rev. D 98, 015034 (2018).

[38] D. Gilman, S. Birrer, A. Nierenberg, T. Treu, X. Du, and A. Benson, Warm dark matter chills out: Constraints on the halo mass function and the free-streaming length of dark matter with 8 quadruple-image strong gravitational lenses, Mon. Not. R. Astron. Soc. 491, 6077 (2020).

[39] A. Garzilli, O. Ruchayskiy, A. Magalich, and A. Boyarsky, How warm is too warm? Towards robust Lyman- $\alpha$ forest bounds on warm dark matter, arXiv:1912.09397.

[40] See Supplemental Material at http://link.aps.org/ supplemental/10.1103/PhysRevLett.125.131301 for further details on the effects of light Co-SIMPs on Neff, the compatibility of the Co-SIMP scenario with the recently observed XENON1T electron-recoil excess, and other potential searches for Co-SIMPs, which includes Refs. [41-97].

[41] S. Pastor, T. Pinto, and G. G. Raffelt, Relic Density of Neutrinos with Primordial Asymmetries, Phys. Rev. Lett. 102, 241302 (2009).

[42] J. Hamann, S. Hannestad, G. G. Raffelt, I. Tamborra, and Y. Y. Y. Wong, Cosmology Seeking Friendship with Sterile Neutrinos, Phys. Rev. Lett. 105, 181301 (2010).

[43] S. Weinberg, Goldstone Bosons as Fractional Cosmic Neutrinos, Phys. Rev. Lett. 110, 241301 (2013).

[44] S. Böser, C. Buck, C. Giunti, J. Lesgourgues, L. Ludhova, S. Mertens, A. Schukraft, and M. Wurm, Status of light sterile neutrino searches, arXiv:1906.01739.

[45] G. Gelmini, S. Palomares-Ruiz, and S. Pascoli, Low Reheating Temperature and the Visible Sterile Neutrino, Phys. Rev. Lett. 93, 081302 (2004).

[46] P. F. de Salas, M. Lattanzi, G. Mangano, G. Miele, S. Pastor, and O. Pisanti, Bounds on very low reheating scenarios after Planck, Phys. Rev. D 92, 123534 (2015).

[47] T. Hasegawa, N. Hiroshima, K. Kohri, R. S. L. Hansen, T. Tram, and S. Hannestad, MeV-scale reheating temperature and thermalization of oscillating neutrinos by radiative and hadronic decays of massive particles, J. Cosmol. Astropart. Phys. 12 (2019) 012.

[48] Abazajian et al., Light sterile neutrinos: A white paper, arXiv:1204.5379.

[49] C. D. Kreisch, F.-Y. Cyr-Racine, and O. Doré, The neutrino puzzle: Anomalies, interactions, and cosmological tensions, Phys. Rev. D 101, 123505 (2020).

[50] A. Berlin and N. Blinov, Thermal Dark Matter Below an MeV, Phys. Rev. Lett. 120, 021801 (2018).

[51] N. Blinov, K. J. Kelly, G. Z. Krnjaic, and S. D. McDermott, Constraining the Self-Interacting Neutrino Interpretation of the Hubble Tension, Phys. Rev. Lett. 123, 191102 (2019).

[52] Planck Collaboration, Planck 2018 results. VI. Cosmological parameters, arXiv:1807.06209.

[53] Xenon1T Collaboration, Observation of excess electronic recoil events in XENON1T, arXiv:2006.09721.

[54] A.E. Robinson, XENON1T observes tritium, arXiv:2006.13278.

[55] I. M. Bloch, A. Caputo, R. Essig, D. Redigolo, M. Sholapurkar, and T. Volansky, Exploring new physics with $\mathrm{O}(\mathrm{keV})$ electron recoils in direct detection experiments, arXiv:2006.14521. 
[56] L. Su, W. Wang, L. Wu, J. M. Yang, and B. Zhu, Atmospheric dark matter from inelastic cosmic ray collision in xenon1T, arXiv:2006.11837.

[57] J. Bramante and N. Song, Electric but not eclectic: Thermal relic dark matter for the XENON1T excess, arXiv: 2006.14089.

[58] M. Du, J. Liang, Z. Liu, V. Q. Tran, and Y. Xue, Onshell mediator dark matter models and the Xenon1T anomaly, arXiv:2006.11949.

[59] D. McKeen, M. Pospelov, and N. Raj, Hydrogen portal to exotic radioactivity, arXiv:2006.15140.

[60] S.-F. Ge, P. Pasquini, and J. Sheng, Solar neutrino scattering with electron into massive sterile neutrino, arXiv:2006.16069.

[61] W. Chao, Y. Gao, and M. Jin, Pseudo-Dirac dark matter in XENON1T, arXiv:2006.16145.

[62] P. Ko and Y. Tang, Semi-annihilating $Z_{3}$ dark matter for XENON1T excess, arXiv:2006.15822.

[63] H. An and D. Yang, Direct detection of freeze-in inelastic dark matter, arXiv:2006.15672.

[64] Y. Gao and T. Li, Lepton number violating electron recoils at XENON1T by the $U(1)_{B-L}$ model with non-standard interactions, arXiv:2006.16192.

[65] H. M. Lee, Exothermic dark matter for XENON1T excess, arXiv:2006.13183.

[66] M. Baryakhtar, A. Berlin, H. Liu, and N. Weiner, Electromagnetic signals of inelastic dark matter scattering, arXiv:2006.13918.

[67] U. K. Dey, T. N. Maity, and T. S. Ray, Prospects of Migdal effect in the explanation of XENON1T electron recoil excess, arXiv:2006.12529.

[68] Y. Jho, J.-C. Park, S. C. Park, and P.-Y. Tseng, Gauged lepton number and cosmic-ray boosted dark matter for the XENON1T excess, arXiv:2006.13910.

[69] C. Gao, J. Liu, L.-T. Wang, X.-P. Wang, W. Xue, and Y.-M. Zhong, Re-examining the solar axion explanation for the XENON1T excess, arXiv:2006.14598.

[70] M. Lindner, Y. Mambrini, T. B. de Melo, and F.S. Queiroz, XENON1T anomaly: A light $Z^{\prime}$, arXiv:2006.14590.

[71] L. Zu, G.-W. Yuan, L. Feng, and Y.-Z. Fan, Mirror dark matter and electronic recoil events in XENON1T, arXiv: 2006.14577.

[72] K. Nakayama and Y. Tang, Gravitational production of hidden photon dark matter in light of the XENON1T excess, arXiv:2006.13159.

[73] H. An, M. Pospelov, J. Pradler, and A. Ritz, New limits on dark photons from solar emission and $\mathrm{keV}$ scale dark matter, arXiv:2006.13929.

[74] A. N. Khan, Can nonstandard neutrino interactions explain the XENON1T spectral excess? arXiv:2006.12887.

[75] R. Primulando, J. Julio, and P. Uttayarat, Collider constraints on a dark matter interpretation of the XENON1T excess, arXiv:2006.13161.

[76] D. Aristizabal Sierra, V. De Romeri, L. J. Flores, and D. K. Papoulias, Light vector mediators facing XENON1T data, arXiv:2006.12457.

[77] J. Buch, M. A. Buen-Abad, J. J. Fan, and J. S. C. Leung, Galactic origin of relativistic bosons and XENON1T excess, arXiv:2006.12488.
[78] Y. Chen, J. Shu, X. Xue, G. Yuan, and Q. Yuan, Sun heated $\mathrm{MeV}$-scale dark matter and the XENON1T electron recoil excess, arXiv:2006.12447.

[79] Q.-H. Cao, R. Ding, and Q.-F. Xiang, Exploring for sub$\mathrm{MeV}$ boosted dark matter from xenon electron direct detection, arXiv:2006.12767.

[80] B. Fornal, P. Sandick, J. Shu, M. Su, and Y. Zhao, Boosted dark matter interpretation of the XENON1T excess, arXiv: 2006.11264.

[81] N. F. Bell, J. B. Dent, B. Dutta, S. Ghosh, J. Kumar, and J. L. Newstead, Explaining the XENON1T excess with luminous dark matter, arXiv:2006.12461.

[82] A. Bally, S. Jana, and A. Trautner, Neutrino self-interactions and XENON1T electron recoil excess, arXiv:2006.11919.

[83] L. Di Luzio, M. Fedele, M. Giannotti, F. Mescia, and E. Nardi, Solar axions cannot explain the XENON1T excess, arXiv:2006.12487.

[84] G. Choi, M. Suzuki, and T. T. Yanagida, XENON1T anomaly and its implication for decaying warm dark matter, arXiv:2006.12348.

[85] K. Harigaya, Y. Nakai, and M. Suzuki, Inelastic dark matter electron scattering and the XENON1T excess, arXiv:2006.11938.

[86] G. Paz, A. A. Petrov, M. Tammaro, and J. Zupan, Shining dark matter in Xenon1T, arXiv:2006.12462.

[87] C. Boehm, D. G. Cerdeno, M. Fairbairn, P. A. N. Machado, and A.C. Vincent, Light new physics in XENON1T, arXiv:2006.11250.

[88] G. Alonso-Álvarez, F. Ertas, J. Jaeckel, F. Kahlhoefer, and L. J. Thormaehlen, Hidden photon dark matter in the light of XENON1T and stellar cooling, arXiv:2006.11243.

[89] F. Takahashi, M. Yamada, and W. Yin, XENON1T anomaly from anomaly-free ALP dark matter and its implications for stellar cooling anomaly, arXiv:2006.10035.

[90] K. Kannike, M. Raidal, H. Veermäe, A. Strumia, and D. Teresi, Dark Matter and the XENON1T electron recoil excess, arXiv:2006.10735.

[91] John R. Lombardi, Hydrogen atom in the momentum representation, Phys. Rev. A 22, 797 (1980).

[92] Dark-Side-50 Collaboration, Constraints on Sub-GeV Dark-Matter-Electron Scattering from the DarkSide-50 Experiment, Phys. Rev. Lett. 121, 111303 (2018).

[93] Dark-Side-50 Collaboration, Low-Mass Dark Matter Search with the DarkSide-50 Experiment, Phys. Rev. Lett. 121, 081307 (2018).

[94] F. Yuan, E. Quataert, and R. Narayan, Nonthermal electrons in radiatively inefficient accretion flow models of Sagittarius A*, Astrophys. J. 598, 301 (2003).

[95] E. Hardy and R. Lasenby, Stellar cooling bounds on new light particles: Plasma mixing effects, J. High Energy Phys. 02 (2017) 033.

[96] G. D. Mack, J. F. Beacom, and G. Bertone, Towards closing the window on strongly interacting dark matter: Far-reaching constraints from Earth's heat flow, Phys. Rev. D 76, 043523 (2007).

[97] X. Chu and C. Garcia-Cely, Self-interacting spin-2 dark matter, Phys. Rev. D 96, 103519 (2017).

[98] C. M. Bender and S. Sarkar, Asymptotic analysis of the Boltzmann equation for dark matter relics, J. Math. Phys. (N.Y.) 53, 103509 (2012). 
[99] M. Hufnagel, K. Schmidt-Hoberg, and S. Wild, BBN constraints on MeV-scale dark sectors. Part II. Electromagnetic decays, J. Cosmol. Astropart. Phys. 11 (2018) 032.

[100] L. Forestell, D. E. Morrissey, and G. White, Limits from BBN on light electromagnetic decays, J. High Energy Phys. 01 (2019) 074.

[101] A. Berlin, N. Blinov, and S. W. Li, Dark sector equilibration during nucleosynthesis, Phys. Rev. D 100, 015038 (2019).

[102] Planck Collaboration, Planck 2018 results. VI. Cosmological parameters, arXiv:1807.06209.

[103] M. Kamionkowski, Indirect detection of WIMPs, arXiv: hep-ph/9403357.

[104] M. Schumann, Direct detection of WIMP dark matter: Concepts and status, J. Phys. G 46, 103003 (2019).

[105] A. Mitridate, M. Redi, J. Smirnov, and A. Strumia, Dark matter as a weakly coupled dark baryon, J. High Energy Phys. 10 (2017) 210.

[106] Y. Grossman, R. Harnik, O. Telem, and Y. Zhang, Selfdestructing dark matter, J. High Energy Phys. 07 (2019) 017.

[107] J. A. Dror, G. Elor, and R. Mcgehee, Direct detection signals from absorption of fermionic dark matter, arXiv: 1905.12635.

[108] J.A. Dror, G. Elor, and R. Mcgehee, Absorption of fermionic dark matter by nuclear targets, arXiv: 1908.10861.

[109] M. Geller and O. Telem, Self destructing atomic DM, arXiv:2001.11514.

[110] Xenon1T Collaboration, Observation of two-neutrino double electron capture in ${ }^{124} \mathrm{Xe}$ with XENON1T, Nature (London) 568, 532 (2019).

[111] BOREXINO Collaboration, Science and technology of BOREXINO: A real time detector for low energy solar neutrinos, Astropart. Phys. 16, 205 (2000).

[112] BOREXINO Collaboration, First simultaneous precision spectroscopy of $p p,{ }^{7} \mathrm{Be}$, and pep solar neutrinos with borexino phase-II, arXiv:1707.09279.

[113] Xenon1T Collaboration, Light Dark Matter Search with Ionization Signals in XENON1T, Phys. Rev. Lett. 123, 251801 (2019).

[114] L. Baudis, Searching for dark matter with the XENON1T Experiment.

[115] DARWIN Collaboration, DARWIN: Towards the ultimate dark matter detector, J. Cosmol. Astropart. Phys. 11 (2016) 017.

[116] KamLAND-Zen Collaboration, Results from KamLANDZen, AIP Conf. Proc. 1666, 170003 (2015).

[117] JUNO Collaboration, Neutrino physics with JUNO, J. Phys. G 43, 030401 (2016).

[118] N. A. Kurinsky, T. C. Yu, Y. Hochberg, and B. Cabrera, Diamond detectors for direct detection of Sub-GeV dark matter, Phys. Rev. D 99, 123005 (2019).

[119] D. M. Mei, G. J. Wang, H. Mei, G. Yang, J. Liu, M.Wagner, R. Panth, K. Kooi, Y. Y. Yang, and W.Z. Wei, Direct detection of $\mathrm{MeV}$-scale dark matter utilizing germanium internal amplification for the charge created by the ionization of impurities, Eur. Phys. J. C 78, 187 (2018).

[120] Y. Hochberg, Y. Kahn, M. Lisanti, K. M. Zurek, A. G. Grushin, R. Ilan, S. M. Griffin, Z.-F. Liu, S. F. Weber, and J. B. Neaton, Detection of sub-MeV dark matter with three-dimensional Dirac materials, Phys. Rev. D 97, 015004 (2018).

[121] Y. Hochberg, M. Pyle, Y. Zhao, and K. M. Zurek, Detecting superlight dark matter with fermi-degenerate materials, J. High Energy Phys. 08 (2016) 057.

[122] S. Knapen, T. Lin, and K. M. Zurek, Light dark matter: Models and constraints, Phys. Rev. D 96, 115021 (2017).

[123] CRESST Collaboration, Results on MeV-scale dark matter from a gram-scale cryogenic calorimeter operated above ground, Eur. Phys. J. C 77, 637 (2017).

[124] F. Ruppin, J. Billard, E. Figueroa-Feliciano, and L. Strigari, Complementarity of dark matter detectors in light of the neutrino background, Phys. Rev. D 90, 083510 (2014).

[125] K. Schutz and K. M. Zurek, Detectability of Light Dark Matter with Super uid Helium, Phys. Rev. Lett. 117, 121302 (2016).

[126] S. A. Hertel, A. Biekert, J. Lin, V. Velan, and D. N. McKinsey, A path to the direct detection of sub-GeV dark matter using calorimetric readout of a super uid ${ }^{4} \mathrm{He}$ target, arXiv:1810.06283.

[127] F. Acanfora, A. Esposito, and A. D. Polosa, Sub-GeV dark matter in super uid He-4: An effective theory approach, Eur. Phys. J. C 79, 549 (2019).

[128] A. Caputo, A. Esposito, and A. D. Polosa, Sub-MeV dark matter and the goldstone modes of Super uid helium, Phys. Rev. D 100, 116007 (2019).

[129] A. Caputo, A. Esposito, E. Geoffray, A. D. Polosa, and S. Sun, Dark Matter, Dark photon and Super uid He-4 from effective field theory, Phys. Lett. B 802, 135258 (2020).

[130] S. Knapen, T. Lin, M. Pyle, and K. M. Zurek, Detection of light dark matter with optical phonons in polar materials, Phys. Lett. B 785, 386 (2018).

[131] S. Griffin, S. Knapen, T. Lin, and K. M. Zurek, Directional detection of light dark matter with polar materials, Phys. Rev. D 98, 115034 (2018).

[132] S. M. Griffin, K. Inzani, T. Trickle, Z. Zhang, and K. M. Zurek, Multi-channel direct detection of light dark matter: Target comparison, arXiv:1910.10716.

[133] Chandra-X Collaboration, Chandra $X$-ray spectroscopic imaging of Sgr A* and the central parsec of the Galaxy, Astrophys. J. 591, 891 (2001).

[134] K. Perez, K. C. Y. Ng, J. F. Beacom, C. Hersh, S. Horiuchi, and R. Krivonos, Almost closing the $\iota$ MSM sterile neutrino dark matter window with NuSTAR, Phys. Rev. D 95, 123002 (2017).

[135] INTEGRAL Collaboration, The athena x-ray integral field unit, arXiv:1807.06092.

[136] M. Baldo and V. Palmisano, Probing post-bounce supernova density profile from neutrino signals, Phys. Rev. C 78, 015807 (2008).

[137] W. DeRocco, P. W. Graham, D. Kasen, G. MarquesTavares, and S. Rajendran, Supernova signals of light dark matter, arXiv: 1905.09284.

[138] A. Ishida, T. Namba, S. Asai, T. Kobayashi, H. Saito, M. Yoshida, K. Tanaka, and A. Yamamoto, New precision measurement of hyperfine splitting of positronium, Phys. Lett. B 734, 338 (2014). 
[139] S. J. Brodsky and R. F. Lebed, Production of the Smallest QED Atom: True Muonium (mu+ mu-), Phys. Rev. Lett. 102, 213401 (2009).

[140] X. Cid Vidal, P. Ilten, J. Plews, B. Shuve, and Y. Soreq, Discovering true muonium at LHCb, Phys. Rev. D 100, 053003 (2019).

[141] J. Kopp, L. Michaels, and J. Smirnov, Loopy constraints on leptophilic dark matter and internal Bremsstrahlung, J. Cosmol. Astropart. Phys. 04 (2014) 022.

[142] P. J. Fox, R. Harnik, J. Kopp, and Y. Tsai, LEP shines light on dark matter, Phys. Rev. D 84, 014028 (2011).

[143] A. Rajaraman, W. Shepherd, T. M. P. Tait, and A. M. Wijangco, LHC bounds on interactions of dark matter, Phys. Rev. D 84, 095013 (2011).

[144] P. J. Fox, R. Harnik, J. Kopp, and Y. Tsai, Missing energy signatures of dark matter at the LHC, Phys. Rev. D 85, 056011 (2012).

[145] N. Daci, I. De Bruyn, S. Lowette, M. H. G. Tytgat, and B. Zaldivar, Simplified SIMPs and the LHC, J. High Energy Phys. 11 (2015) 108.
[146] C. V. Cappiello, K. C. Y. Ng, and J. F. Beacom, Reverse direct detection: Cosmic ray scattering with light dark matter, Phys. Rev. D 99, 063004 (2019).

[147] T. Bringmann and M. Pospelov, Novel Direct Detection Constraints on Light Dark Matter, Phys. Rev. Lett. 122, 171801 (2019).

[148] Y. Ema, F. Sala, and R. Sato, Light Dark Matter at Neutrino Experiments, Phys. Rev. Lett. 122, 181802 (2019).

[149] C. Cappiello and J. F. Beacom, Strong new limits on light dark matter from neutrino experiments, arXiv:1906.11283.

[150] J. B. Dent, B. Dutta, J. L. Newstead, and I. M. Shoemaker, Bounds on cosmic ray-boosted dark matter in simplified models and its corresponding neutrino-floor, arXiv: 1907.03782.

Correction: The captions and images for Figures 3 and 4 were positioned improperly in the final layout and have been fixed. 